\section{POS0368 CITRULLINATION INDUCES EPIGENETIC MEMORY OF THE INNATE IMMUNE SYSTEM}

K. Laskari ${ }^{1}$, S. Sabu ${ }^{1}$, O. Distler ${ }^{1}$, M. Neidhart ${ }^{1}$, E. Karouzakis ${ }^{1,2} .{ }^{1}$ University Hospital Zurich, University of Zurich, Department of Rheumatology, Center of Experimental Rheumatology, Zurich, Switzerland; ${ }^{2}$ Galapagos, Translational Medicine, Basel, Switzerland

Background: During trained immunity, monocytes and macrophages undergo a functional and transcriptional reprogramming toward activation, which is induced by a priming stimulus and results in enhanced responsiveness to subsequent triggers. Monocytes from patients with rheumatoid arthritis (RA) display features consistent with a trained immunity phenotype. Citrullinated proteins as citrullinated vimentin (c-vimentin), which function as damage-associated patterns in RA, may be implicated in the process of trained immunity.

Objectives: We aimed to investigate if c-vimentin induces trained immunity in vitro in healthy individuals.

Methods: Monocytes were isolated from the peripheral blood (EDTA blood, $\mathrm{n}=22$; buffy coats, $\mathrm{n}=6$ ) from healthy donors by Ficoll-paque centrifugation and negative selection using CD3/CD19/CD56 magnetic beads. The cells were stimulated with c-vimentin $(0.1 \mu \mathrm{g} / \mathrm{ml})$ for $24 \mathrm{~h}$ and re-stimulated 5 days later with the lipopolysaccharide of E.coli (LPS) $(10 \mathrm{ng} / \mathrm{ml})$. Protein as well as lactate release were estimated in cell culture supernatants at day 6 by ELISA. RT-PCR and/or Western Blotting were applied to measure mRNA and/ or protein expression. The Ligand-receptor glycocapture technology LRC-TRiCEPS was used to identify candidate cell surface targets of c-vimentin. The methylation of histone $\mathrm{H} 3$ at lysine $4(\mathrm{H} 3 \mathrm{~K} 4)$ was examined by chromatin immunoprecipitation.

Results: Priming with citrullinated vimentin induced training in human monocytes, as suggested by the significantly increased levels of secreted interleukin-6 (IL-6), upon restimulation with LPS (1.29-fold increase, $n=22, p<0.001$ ). Likewise, the release of chemokines CXCL1 and CCL20/Macrophage Inflammatory Protein 3a was significantly increased (1.81-fold and 2.32-fold increase, respectively, $\mathrm{n}=14$, both $\mathrm{p}<0.001$ ). LRC-TRiCEPS enabled the identification of STING cell surface receptor for the ligand $\mathrm{c}$-vimentin. Indeed, c-vimentin induced activation of TBK1, which is implicated in the STING signaling pathway, by phosphorylation, while STING inhibition with the covalent small molecule H151 $(2 \mu \mathrm{M})$ abolished this effect. Besides, $\mathrm{H} 151$ inhibited trained immunity by decreasing IL-6 release and expression (1.61-fold and 1.93-fold decrease, respectively, $n=5$ ). Trained monocytes also displayed high lactate production (primed vs. unprimed cells, $\mathrm{n}=9, \mathrm{p}=0.004$ ), reflecting a shift in metabolism with an increase in glycolysis. By inhibiting the metabolic pathway of glycolysis by 2-deoxyglucose (11 $\mathrm{mM})$, the induction of trained immunity could be counteracted (5.32-fold decrease in IL-6 release, $n=7, p=0.016)$. Finally, c-vimentin induced H3K4 methylation with increased levels of this mark in the promoter of the IL- 6 gene. By modulating the function of epigenetic enzymes with methylthioadenosine ( $1 \mathrm{mM})$, which specifically inhibits histone methyltransferase, trained immunity was reversed (8.43-fold decrease in IL-6 release, $n=6, p=0.031$ ).

Conclusion: Citrullinated vimentin induces epigenetic modifications and metabolic changes in monocytes, probably through a STING and TBK1-dependent activation, resulting in enhanced cytokine and chemokine production upon restimulation. Inhibition of the STING signaling pathway may be a novel therapeutic target for myeloid activation in RA.

Disclosure of Interests: None declared

DOI: 10.1136/annrheumdis-2021-eular.3302

\section{POS0369 \\ ELEVATED EXPRESSION OF TIM-3 ON NEUTROPHILS CORRELATES WITH DISEASE ACTIVITY AND SEVERITY OF ANKYLOSING SPONDYLITIS}

X. Huang ${ }^{1}$, T. W. Li ${ }^{1}$, J. Chen ${ }^{1}$, Z. Huang ${ }^{1}$, S. Chen ${ }^{1}$, X. Guo' ${ }^{1}{ }^{1}$ Guangdong Second Provincial General Hospital, Department of Rheumatology and Immunology, Guangzhou, China

Background: Ankylosing spondylitis (AS) is a type of common, chronic inflammatory disease that compromises the axial skeleton and sacroiliac joints, causing inflammatory low back pain and progressive spinal stiffness, over time some patients develop spinal immobility and ankylosis which can lead to a decrease in quality of life. The last few decades, evidence has clearly indicated that neutrophil also plays key roles in the progression of AS. However, the immunomodulatory roles and mechanisms of neutrophils in AS are poorly understood. T-cell immunoglobulin and mucin domain-containing protein 3 (Tim-3) has been reported as an important regulatory molecule, expressed and regulated on different innate immune cells, plays a pivotal role in several autoimmunity diseases. Recent study indicates that Tim3 is also expressed on neutrophils. However, the frequency and roles of Tim3-expressing neutrophils in AS was not clear.

Objectives: In this study, we investigated the expression of Tim3 on neutrophils in AS patients and explored the correlation between the level of Tim3-expressing neutrophils and the disease activity and severity of AS.

Methods: Patients with AS were recruited from Guangdong Second Provincial General Hospital $(n=62)$. Age/sex-matched volunteers as Healthy controls $(\mathrm{HC})$ $(n=39)$. The medical history, clinical manifestations, physical examination, laboratory measurements were recorded. The expression of costimulatory molecules including programmed death 1 (PD-1), Tim-3 on neutrophils were determined by flow cytometry. The mRNA expression of PD-1 and Tim-3 was determined by real-time PCR. The levels of Tim3-expressing neutrophils in AS patients were further analyzed for their correlation with the markers of inflammation such as ESR,CRP,WBC and neutrophil count(NE), as well as disease activity and severity of AS. The expression of Tim3 on neutrophils was monitored during the course of treatment (4 weeks).

Results: The expression of Tim3 on neutrophils in patients with AS was increased compared to the HC (Figure 1A). However, significant difference was observed in the frequency of $\mathrm{PD}$-1-expressing neutrophils between AS patients and HC (Figure 1B). The expression analysis of Tim-3 mRNA, but not PD-1, confirmed the results obtained from flow cytometry (Figure 1C). The level of Tim3-expressing neutrophils in patients with AS showed an positive correlation with ESR, CRP and ASAS-endorsed disease activity score (ASDAS) (Figure 1D). Moreover the frequency of Tim3-expressing neutrophils in active patients(ASDAS $\geq 1.3$ ) was increased as compare with the inactive patients (ASDAS 1.3 ) (Figure 1E). As shown in Figure 1F, the frequency of Tim3-expressing neutrophils decreased after the treatment.

A

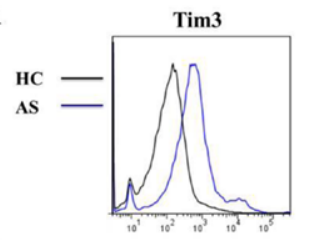

B

PD-1
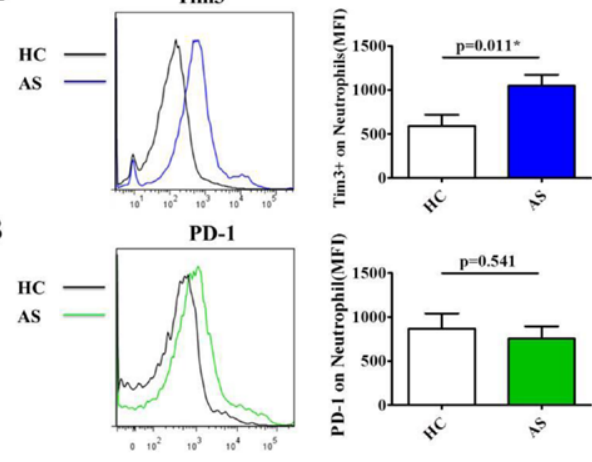

C
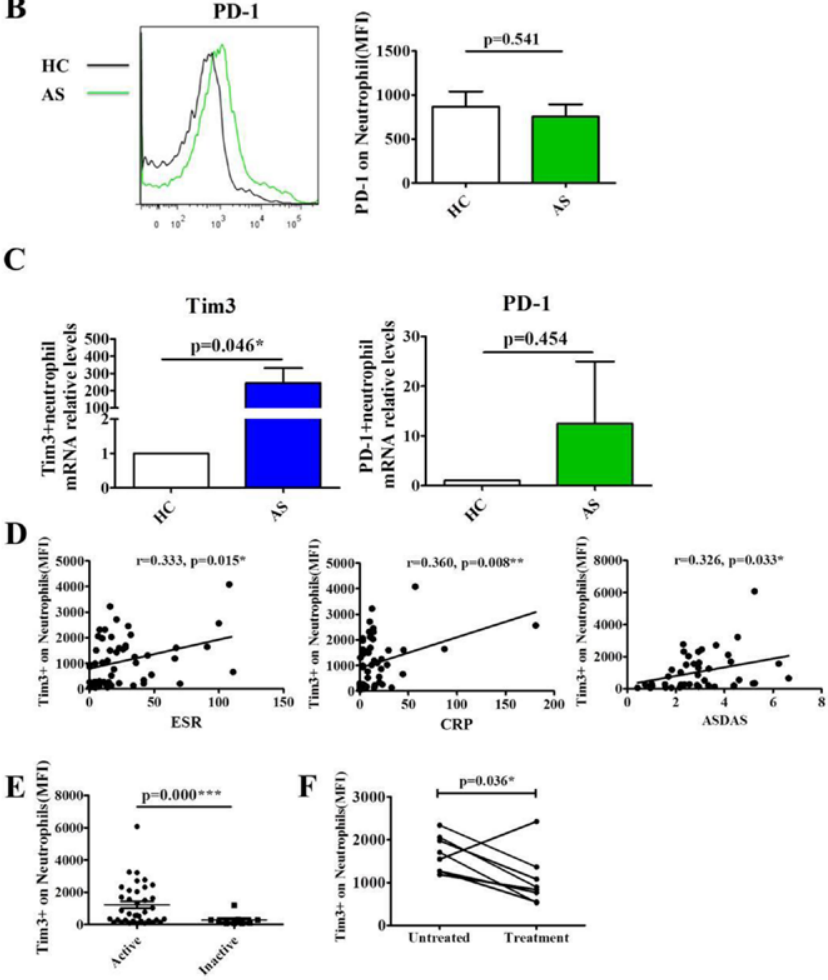

Figure 1. (A,B)The expression of Tim3 and PD-1 on neutrophils in AS and $\mathrm{HC}$ were deter mined by flow cytometry.(C) The expression of Tim3 and PD-1 on neutrophils in AS and HC were determined by RT-PCR.(D)The correction between Tim3-expressing neutrophils and ESR,CRP,ASDAS.(E) The expression of Tim3 on neutrophils in active and inactive patients.(F) Influence of treatment on the frequency of Tim3-expressing neutrophils. 